\title{
LATERAL DEFLECTIONS OF WEBS IN AIR-FLOTATION OVENS
}

\author{
by \\ P. M. Moretti \\ Oklahoma State University \\ USA
}

\begin{abstract}
A long web span supported by many, regularly spaced, alternating air-bars is studied. The focus is on the lateral forces on the web due to the interaction of lateral curvature with out-of-plane deflections. The effect of stretching of the elastic web material is included, and the effect of high web speed is handled by distinguishing between the tension in the material $T_{m a t}$ and the apparent tension $T_{a p p}=T_{m a t}-\mu v^{2}$.

The governing Partial Differential Equations for a continuous representation of the web's lateral deflection, stability, and control is developed for both straight and cambered webs. The dimensionless parameters for web-tension effect, web-camber effect, and stretching effect are identified.

The influence of tilted air-bars is studied, towards developing a control mechanism to compensate for camber.
\end{abstract}

\section{NOMENCLATURE}

$A$ cross-sectional area of web (thickness $\times$ width $b$ )

$b$ width of web

$E A$ extensional stiffness of web undulating over air supports

$E I$ lateral bending stiffness of air-supported web

$\mathfrak{F}$ functional dependence of pad pressure $P$ on flying height $\left(Z-z_{b a r}\right)$

$k$ extensional spring constant of web span, per unit width

$L \quad$ Length of entire web-span within air-flotation oven

$\ell$ spacing of air-support bars, half-wavelength of sinusoidal shape

$p$ local gage pressure between air-support bar and web

$P$ maximum gage pressure between air-support bar and web

$S$ length of web path in air-flotation oven, $>L$ because of waviness

$s$ length of one-half wave of web, $>\ell$

$T_{\text {mat }}$ actual tension in the web material, per unit width

$T_{a p p}$ web tension apparent to a dancer-roller, per unit width

$v$ machine-direction velocity of web 


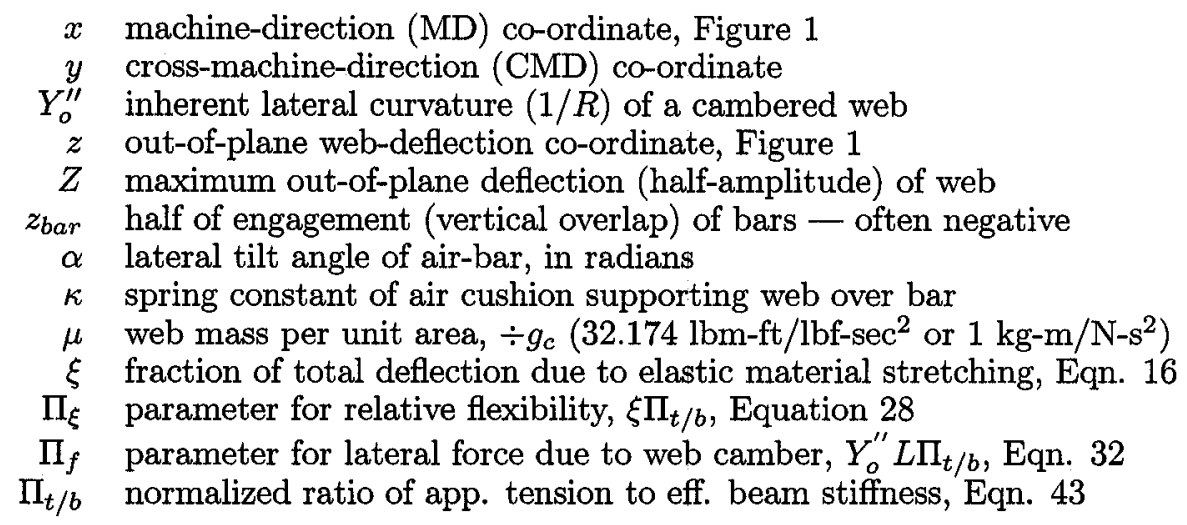

\section{INTRODUCTION}

In the operation of flotation ovens, lateral deflections have been observed. This is an important problem, because there is a need for longer air-support ovens to accomplish drying of water-based coatings, yet the length of the ovens is limited by our ability to control lateral excursions of the web. For the purpose of analytical study of an oven containing many similar elements. (i.e. regular undulations between bottom air-bars alternating with top air-bars), the entire length of the web within the oven may be represented as a single continuum element with continuous-beam properties. These averaged properties depend on the engagement geometry of the alternating air-bars, the air-cushion characteristics of each bar, the web tension, and the web elasticity.

\section{BACKGROUND}

Tests on lateral deflection in air-support conveyance by Ron Swanson [1], and further tests on extensional resilience of an air-floated web by Ron Swanson, YoungBae Chang, and Peter Moretti [2], led to the following observations:

- Lateral forces required to deflect the web are small.

- Webs in air-suspension ovens tend to diverge to one side or the other.

- Web camber aggravates the lateral divergence.

- Correction/control by tilting air-flotation bars yields small improvement.

\section{MID-SPAN BEHAVIOR}

As we pointed out in an exploratory paper [3], there are several different factors at play in the lateral deflection of a web in an air-flotation oven:

- A web on air-support bars is supported by the air cushions in a "springy" fashion, permitting deflections in the out-of-plane (usually vertical) direction. Because of the accordion-like undulations of the web, these potential deflections cause it to be less stiff with respect to longitudinal (MD) extension as well, which in turn yields less beam stiffness in the lateral direction (CMD), so that small lateral forces can result in significant deflection to one side or the other. Lateral stiffness can be improved by using low-flying-height airfoil bars giving "stiff" support, or by using high tension, or by using shallow undulations. 
- When a perturbation causes the mid-span of the web to deflect to one side, and that side of the web curves outward, the inside of the curve floats higher off the air supports, and the outside cinches down tighter on the supports, so that the web tilts over each pad. As a result the normal (lift) vector of the pressure force over each pad acquires a lateral force component towards the outside of the curve, tending to increase the lateral curvature further-a destabilizing effect inherent in this geometry. (A secondary effect is a small cross-flow drag force in the opposite direction.)

- Web camber is one of the perturbations which initiates web divergence. Furthermore, when a web has camber, the longer edge of the web will tend to float higher off the air supports, so that it the normal vector of the pressure force will tend to push the web towards the side with the shorter edge.

- Since the tilt of the web, not of the air-bar, determines the lateral component of the lift vector, tilting air-bars has only a modest effect on lateral dynamicsbut it does introduce a secondary effect of biased lateral pressure distribution, with consequences for both the tension profile and the lateral drag forces.

In subsequent sections we will quantify these effects.

\section{EXTENSIONAL STIFFNESS}

\section{Sinusoidal geometry}

We will approximate a symmetrical web path by the first term of a Fourier series representation

$$
\begin{aligned}
z_{(x)} & =Z_{1} \sin \frac{\pi x}{\ell}-Z_{3} \sin \frac{3 \pi x}{\ell}+\ldots \\
& \cong Z \sin \frac{\pi x}{\ell}
\end{aligned}
$$

where $\ell$ is the spacing between (alternating) air-bars and $Z$ is the half-amplitude of the undulation. The equilibrium relationship between apparent tension-per-unitwidth $T_{a p p} \triangleq\left(T_{m a t}-\mu v^{2}\right)$ and the gage pressure $p$ acting on the flexible web, as shown in Figure 2, is

$$
\begin{aligned}
p_{(x)} & =-T_{a p p} \cdot \frac{\partial^{2} z}{\partial x^{2}} \\
& =T_{a p p} \cdot Z\left(\frac{\pi}{\ell}\right)^{2} \sin \frac{\pi x}{\ell} \\
& =P \sin \frac{\pi x}{\ell} \\
\text { where } P & =T_{a p p} \cdot Z\left(\frac{\pi}{\ell}\right)^{2}
\end{aligned}
$$

for an average pressure of $2 P / \pi$, and a total lift of $2 P b \ell / \pi=2 b T_{a p p} Z \pi / \ell$, over each half-sine-wave opposing an air-bar. The constant difference $\mu v^{2}$ between the apparent tension $T_{\text {app }}$ measured from roller reaction forces, and the material tension $T_{m a t}$ observed in the stretching of the web material, is small in most plastic-coating applications; but we will maintain the distinction in order to make our equations applicable to high-speed webs as well. 
This gives us one relationship between air-pad pressure, deflection-amplitude, and tension; we need a second relationship to get useful stiffness results.

\section{Pad pressure vs. flying height}

In a long series of experiments carried out in the Web Handling Research Center at Oklahoma State University by Pinnamaraju [4], Perdue [5], and Nisankararao [6], the pressure field between an air-bar and a flat plate was measured, and integrated to obtain the total lift generated by an air-bar. The lift was shown to be a function of the spacing between air-bar and plate (flying height). The shape of the plotted function, shown in an earlier paper [3], depends on the design of the air bar. A theoretical analysis based on ground-effect hovercraft theory was developed by Young-Bae Chang $[7,8]$ for symmetrical "pressure-pad" bars, matching the experimental results. Some asymmetrical designs, notably "airfoil" or "Coanda" bars, give "stiffer" support to the plate, showing more rapid increase of lift with decrease in flying height.

Although our flexing web differs somewhat from the flat plate, and the simple pressure distribution assumed for this analysis is also different, we can at least conclude that the nominal pad-pressure $P$ is a somewhat similar function of the flying height. The nominal flying height is $\left(Z-z_{b a r}\right)$, where $Z$ is the sinusoidal amplitude of the web, and $z_{b a r}$ is the distance by which each air-bar intrudes past a straight line through the oven-a positive number if the alternating air-bars overlap, negative if they do not cross the central plane. We can generically represent this functional relationship as

$$
P=\mathfrak{F}\left(Z, z_{b a r}\right) \cong \mathfrak{F}\left(Z-z_{b a r}\right)
$$

For small deflections, it may be convenient to linearize this relationship near the design point with an air-cushion "spring-constant" $\kappa \triangleq-d P / d Z$, so that the pressure is

$$
\begin{aligned}
\mathfrak{F} & \approx P_{\text {design }}-\kappa\left(Z-Z_{\text {design }}\right) \\
\frac{d \mathfrak{F}}{d Z} & \approx-\kappa
\end{aligned}
$$

where $\kappa$ is typically positive for stable air-bars (i.e. the pressure drops when the flying height increases). Alternatively, for larger deflections, it may be better to fit a hyperbola to the function, leading to the expression

$$
\begin{aligned}
\mathfrak{F} & \cong P_{\text {design }} \times \frac{\left(Z_{\text {design }}-z_{\text {bar }}\right)}{\left(Z-z_{\text {bar }}\right)} \\
\frac{d \mathfrak{F}}{d Z} & =-P_{\text {design }} \times \frac{\left(Z_{\text {design }}-z_{\text {bar }}\right)}{\left(Z-z_{\text {bar }}\right)^{2}}
\end{aligned}
$$

If the relationship between pressure and flying height has not been measured for a particular air-bar, it may be calculated on the basis of hovercraft theory. The applicable ground-effect analyses $[7,8]$ generally lead to a basic form

$$
\mathfrak{F}=P_{o}\left(1-e^{\frac{- \text { constapt }}{\mathcal{Z}-x_{\text {bar }}}}\right)
$$

plus some added complexity relating to the difference between the nominal flying height and the actual distance from the edge of the slot to the corresponding location on the web. 
Any of these representations of $\mathfrak{F}\left(Z-z_{\text {bar }}\right)$ can be inserted into Equation 2, solved for tension

$$
T_{a p p}=\left(\frac{\ell}{\pi}\right)^{2} \frac{\mathfrak{F}}{Z}
$$

and differentiated to obtain

$$
\frac{d T}{d Z}=\frac{-1}{Z}\left[T_{a p p}-\left(\frac{\ell}{\pi}\right)^{2} \frac{d \mathfrak{F}}{d Z}\right]
$$

This gives us a second relationship between tension, deflection-amplitude, and the pressure function.

\section{Extensibility}

The path length $s$ of the web for each machine-length $\ell$ (and the overall web path length $S$ for the overall machine length $L$ ) is

$$
s=\int_{0}^{\ell} \sqrt{1+a^{2} \cos ^{2} \frac{\pi x}{\ell}} d x
$$

where $a \triangleq \pi Z / \ell$. For $a<1$, we can use the series solution

$$
\frac{S}{L}=\frac{s}{\ell}=1+\frac{1}{4} a^{2}-\frac{3}{64} a^{4}+\frac{5}{256} a^{6}-\ldots
$$

which shows that, when $a^{2} \ll 1$,

$$
\begin{aligned}
\frac{s}{\ell} & \cong 1+\frac{1}{4} a^{2}=1+\frac{\pi^{2} Z^{2}}{4 \ell^{2}} \\
d s & \cong \frac{\pi^{2} Z}{2 \ell} d Z \\
d S & \cong \frac{\pi^{2} Z}{2 L} d Z
\end{aligned}
$$

Inverting the $T-Z$ Equation 8 above,

$$
\frac{d Z}{d T}=\frac{-Z}{T_{a p p}-\left(\frac{\ell}{\pi}\right)^{2} \frac{d \mathcal{I}}{d Z}}
$$

we combine it with Equation 11 to eliminate $d Z$ in the equation for $d S$, and obtain

$$
k_{a i r} \triangleq \frac{-d T}{d S} \cong \frac{2 L}{\pi^{2} Z^{2}}\left[T_{a p p}-\left(\frac{\ell}{\pi}\right)^{2} \frac{d \mathfrak{F}}{d Z}\right]
$$

Since a lengthened path $d S$ shows up as an apparent shortening of the web as seen from the ends of the oven, $d T / d S$ is the negative of $k_{a i r}$, the extensional spring constant per-unit-width of the web in extension, due to the air-cushioning.

In general, observations [2] confirm that extensional stiffness increases if tension is increased; in one test, when the weight attached to the end of a stationary web in an air-support oven was doubled and tripled, the natural frequency of extensional oscillations changed only slightly, indicating that the spring constant approximately doubled and tripled as well. 
The extensional spring constant $k_{a i r}$ obtained in Equation 13 accounts only for air-cushioned out-of-plane deflection; the deflection due to the material's elastic stretching

$$
k_{\text {mat }}=\frac{E_{\text {mat }} A}{L b}
$$

must be added to that, so that the total effective spring constant is

$$
\frac{1}{k_{e f f}}=\frac{1}{k_{a i r}}+\frac{1}{k_{m a t}}
$$

In the vicinity of any particular operation point, the total extension due to an additional increment of tension is composed of both an air-cushion-geometry component and an elastic-stretching component. The fraction of the additional extension due to the web material's elastic stretching, $\xi$, is

$$
\text { stretch-ratio: } \xi \triangleq \frac{1 / k_{\text {mat }}}{1 / k_{\text {eff }}}=\frac{k_{\text {air }}}{k_{\text {mat }}+k_{\text {air }}}
$$

and its complement $(1-\xi)$ is the fraction due to the air-cushion effects:

$$
(1-\xi)=\frac{1 / k_{a i r}}{1 / k_{e f f}}=\frac{k_{m a t}}{k_{m a t}+k_{a i r}}
$$

If the air-bar characteristics are not available, these extensional spring constants can be obtained experimentally. The $k_{m a t}$ can be measured by pulling on the end of the stationary web when the air is turned off, and observing the displacement. The $k_{e f f}$ can be obtained the same way, but while the air is on, and $\xi$ determined from Equation 16. Then $k_{a i r}$ can be computed by solving for it in the Equation 15 and the effective air-cushion constant $\kappa \triangleq-d \mathfrak{F} / d Z$ of the air-support bars backed out from Equation 13.

\section{LATERAL EFFECTS}

\section{Lateral bending stiffness}

J.J. Shelton [9] treated a web as a flat beam, resisting lateral curvature with a lateral bending moment $E I \cdot y^{\prime \prime}$. The effective value of Young's modulus for a web of width $b$ and cross-sectional area $A$ is obtained from

$$
E_{e f f} A=L b k_{e f f}
$$

and

$$
I=A \cdot b^{2} / 12
$$

so that

$$
E_{e f f} I=\frac{L b^{3}}{12} k_{e f f}
$$

where $k_{\text {eff }}$ may be obtained from Equations 13, 14, and 15, or directly from an extensional test with the air-supply turned on.

Thus we can obtain the effective bending stiffness from operating parameters and material properties. 


\section{Lateral moments}

The complementary way of viewing the lateral bending stiffness is to consider lateral curvature. Like extension, bending has both an elastic-material and an aircushion component: The total lateral curvature is the sum of two components, the apparent curvature (as seen from a top view) due to the geometric coupling of out-of-plane deflections with the projected path, $y_{a i r}^{\prime \prime}$, plus the curvature due to differential stretching of the web material by the lateral bending moment, $y_{\text {mat }}^{\prime \prime}$ :

$$
\begin{aligned}
y_{\text {total }}^{\prime \prime} & =y_{\text {mat }}^{\prime \prime}+y_{\text {air }}^{\prime \prime} \\
y_{\text {mat }}^{\prime \prime} & =\xi \cdot y_{\text {total }}^{\prime \prime}=\frac{12 \times \text { Moment }}{L b^{3} k_{\text {mat }}} \\
y_{\text {air }}^{\prime \prime} & =(1-\xi) \cdot y_{\text {total }}^{\prime \prime}=\frac{12 \times \text { Moment }}{L b^{3} k_{\text {air }}} \\
y_{\text {total }}^{\prime \prime} & =\frac{12 \times \text { Moment }}{L b^{3} k_{\text {eff }}}
\end{aligned}
$$

The apparent lateral bending component $y_{\text {air }}^{\prime \prime}$, averaged over multiple elements of air-support, requires the path of one edge of the web to become longer relative to the other edge, by an amount $b \ell \cdot y_{a i r}^{\prime \prime}$, where $b$ is the width of the web and $\ell$ is the length under consideration. A change in length implies a change in the out-of-plane amplitude $Z$, as derived in Equation 11 above:

$$
\begin{aligned}
\frac{d s}{d y} & \cong \frac{\pi^{2} Z}{2 \ell} \cdot \frac{d Z}{d y} \\
\frac{d Z}{d y} & \cong \frac{2 \ell}{\pi^{2} Z} \cdot \frac{d s}{d y} \\
& \cong \frac{2 \ell^{2}}{\pi^{2} Z} \cdot y_{a i r}^{\prime \prime}
\end{aligned}
$$

showing the tilting of the maximum amplitude $Z$ resulting from the apparent lateral bending $y_{a i r}^{\prime \prime}$. Combining this with the $T-Z$ relationship of Equation 8, we find that the tension profile's $d T / d y$ is a function of the nominal tilt $d Z / d y$.

$$
\begin{aligned}
y_{a i r}^{\prime \prime} & =\frac{-\pi^{2} Z^{2}}{2 \ell^{2}\left[T_{a p p}-\left(\frac{\ell}{\pi}\right)^{2} \frac{d \xi}{d Z}\right]} \cdot \frac{d T}{d y} \\
\text { Moment } & =\frac{b^{3}}{12} \cdot \frac{d T}{d y}=\left[\frac{b^{3}}{6}\left(\frac{\ell}{\pi Z}\right)^{2}\left[T_{a p p}-\left(\frac{\ell}{\pi}\right)^{2} \frac{d \mathfrak{F}}{d Z}\right]\right] \cdot y_{a i r}^{\prime \prime}
\end{aligned}
$$

so that the lateral bending moment is governed by the tilt over the air-bars.

\section{Lateral forces}

The lateral curvature component $y_{a i r}^{\prime \prime}$ due to the geometric effects of the web undulating over and under air-support bars has been described in an earlier paper [3] and results in Equation 22, which shows the relationship between $y_{a i r}^{\prime \prime}$ and tilting of the maximum amplitude $Z$. Along the length of the web, the tilt of $z$ is

$$
\frac{\partial z}{\partial y}=\frac{d Z}{d y} \sin \left(\frac{\pi x}{\ell}\right)=\frac{2 \ell^{2}}{\pi^{2} Z} \sin \left(\frac{\pi x}{\ell}\right) \cdot y_{a i r}^{\prime \prime}
$$


Combining this with the pressure obtained in Equation 2, the lateral component of the pressure force on the sinusoidal web is, on the average,

$$
\begin{aligned}
\left|\frac{F_{y}}{\ell}\right| & =\frac{b}{\ell} \int_{0}^{\ell} p \cdot \frac{\partial z}{\partial y} \cdot d x \\
& =\frac{b}{\ell} \int_{0}^{\ell} P \sin \frac{\pi x}{\ell} \cdot \frac{2 \ell^{2}}{\pi^{2} Z} \sin \left(\frac{\pi x}{\ell}\right) \cdot y_{a i r}^{\prime \prime} \cdot d x \\
& =b T_{a p p} \cdot y_{a i r}^{\prime \prime}
\end{aligned}
$$

This establishes that the air-supports produce a lateral force, proportional to that portion of the total lateral curvature $y^{\prime \prime}$ which is due to geometric effects: $y_{\text {air }}^{\prime \prime}=$ $(1-\xi) y_{\text {total }}^{\prime \prime}$

\section{CONTINUUM P.D.E.}

\section{Straight webs}

Introducing this lateral force $F_{y}=-b T \cdot y^{\prime \prime}$ into the partial differential equation for purely lateral motion we obtain for the total deflection $y$

$$
\begin{aligned}
\mu \ddot{y}+2 \mu v \ddot{y}^{\prime}-b T_{a p p} \cdot y^{\prime \prime}+E_{e f f} I y^{\prime \prime \prime \prime} & =-b T_{a p p} \cdot y_{a i r}^{\prime \prime} \\
& =-b T_{a p p} \cdot(1-\xi) y^{\prime \prime} \\
\mu \ddot{y}+2 \mu v \dot{y}^{\prime}-b T_{a p p} \cdot \xi \cdot y^{\prime \prime}+E_{e f f} I y^{\prime \prime \prime \prime} & =0
\end{aligned}
$$

observing that the lateral force from tilting tends to cancel the straightening effect of tension, leaving only the fraction $\xi$ of the hoped-for benefit. Note that this cancellation is independent of the form of the function $\mathfrak{F}$, whether it is linear, or hyperbolic, or based on the ground-effect model.

For the equilibrium solution we can leave out the first two terms, which contain time derivatives, and rearrange the remaining terms to obtain the equilibrium equation

$$
E_{e f f} I y^{\prime \prime \prime \prime}-\xi b T_{a p p} y^{\prime \prime}=0
$$

where $E_{\text {eff }} I$ is the much-reduced value obtained in Equation 20 above, $T_{a p p} \triangleq$ $\left(T_{m a t}-\mu v^{2}\right)$, and $\xi$ is the fraction of the web flexibility due to material elasticity. The apparent lateral stiffness is small if $\xi$ is small; $\xi$ will have a larger value if the web flexibility due to the air-cushioning can be kept small by means of small amplitudes $Z$ and high stiffness $d \mathcal{F} / d Z$. However, the equation is basically stable if $T_{a p p}$ is positive (it goes unstable for negative values exceeding Euler's buckling load).

If we normalize the dimensions $x$ and $y$ by dividing them by the span-length $L$, the dimensionless form of the equilibrium equation yields the independent parameter

$$
\text { stretch-parameter: } \Pi_{\xi} \triangleq \xi b T_{a p p} L^{2} / E_{\text {eff }} I
$$

to govern the solutions.

\section{Cambered webs}

When the web is cambered with an inherent lateral curvature $Y_{o}^{\prime \prime}$, two terms in our equations are modified: the beam stiffness term becomes zero when the web 
follows its natural camber, and the lateral force is zero when the web is level:

$$
\begin{aligned}
\mu \ddot{y}+2 \mu v_{w e b} \ddot{y}^{\prime}-b T_{a p p} y^{\prime \prime}+\frac{\partial^{2}}{\partial x^{2}} E_{e f f} I\left(y^{\prime \prime}-Y_{o}^{\prime \prime}\right) & =b T_{a p p} \cdot\left(Y_{o}^{\prime \prime}-y_{a i r}^{\prime \prime}\right) \\
& =b T_{a p p} Y_{o}^{\prime \prime}-b T_{a p p}(1-\xi) y^{\prime \prime} \\
\ddot{y}+2 \mu v_{w e b} \ddot{y}^{\prime}-b T_{a p p} \cdot \xi \cdot y^{\prime \prime}+\frac{\partial^{2}}{\partial x^{2}} E_{e f f} I & \left(y^{\prime \prime}-Y_{o}^{\prime \prime}\right)=b T_{a p p} \cdot Y_{o}^{\prime \prime}
\end{aligned}
$$

If the camber is constant along the length of the web, this simplifies to

$$
\mu \ddot{y}+2 \mu v_{w e b} \ddot{y}^{\prime}-b T_{a p p} \cdot \xi \cdot y^{\prime \prime}+E_{e f f} I y^{\prime \prime \prime \prime}=b T_{a p p} Y_{o}^{\prime \prime}
$$

so that our governing equation has acquired a constant lateral force-per-unit-length $f_{y}=b T Y_{o}^{\prime \prime}$, acting towards the concave side of the web camber. The equilibrium equation is

$$
E_{\text {eff }} I y^{\prime \prime \prime \prime}-b T_{a p p} \cdot \xi \cdot y^{\prime \prime}=b T_{a p p} Y_{o}^{\prime \prime}
$$

Because of the reduced effective beam stiffness $E_{\text {eff }} I$ and the considerable length of most air-flotation ovens, the effect of any lateral load $f_{y}$ is considerable.

If we normalize the dimensions $x$ and $y$ by dividing them by the span-length $L$, the dimensionless form of the equilibrium equation yields the additional independent parameter

$$
\text { camber-parameter: } \Pi_{f} \triangleq b T_{a p p} Y_{o}^{\prime \prime} L^{3} / E_{e f f} I
$$

\section{Boundary conditions}

At the entry to the span, the displacement $y_{(x=0)}$ and the slope $y_{(x=0)}^{\prime}$ are given; we establish the origin and alignment of the co-ordinates to make both of them zero:

$$
\begin{aligned}
& y_{(x=0)}=0 \\
& y_{(x=0)}^{\prime}=0
\end{aligned}
$$

In practice, the exit roller will be guided to maintain the exit displacement at

$$
y_{(x=L)}=0
$$

by slanting the roller within the plane of the web, so that there will be an exit angle $y_{(x=L)}^{\prime}=\theta_{e x}$. The fourth boundary condition is more difficult, especially in the presence of web camber, as discussed by Ron Swanson [1].

If roller traction is good and moments are moderate, equilibrium also requires that there must be a sufficient moment at the exit to insure that the both sides of the web have the same length as they feed through the roller, so that we have Swanson's moderate-span full-width-traction boundary condition

$$
y_{(x=L)}^{\prime \prime}=0
$$

This condition would break down in the presence of large moments at the exit if the tension were not sufficient to maintain traction across the entire width of the roller, and we would end up with Swanson's long-span partial-slip boundary condition $y_{(x=L)}^{\prime \prime} \approx Y_{o}^{\prime \prime}$, indicating that the unequal-length edges would manage to slip through. 


\section{Equilibrium solution}

In terms of the parameters from Equations 28 and 32, our governing Equation 31 is

$$
y^{\prime \prime \prime \prime}-\frac{\Pi_{\xi}}{L^{2}} y^{\prime \prime}=\frac{\Pi_{f}}{L^{3}}
$$

If we apply Swanson's full-width-traction exit boundary condition $y_{(x=L)}^{\prime \prime}=0$, so that both sides of the web feed through the roller evenly, the solution is

$$
\frac{y}{L}=C_{o}+C_{1}\left(\frac{x}{L}\right)+A \sin \left(\sqrt{\Pi_{\xi}} \frac{x}{L}\right)+B \cos \left(\sqrt{\Pi_{\xi}} \frac{x}{L}\right)+\frac{\Pi_{f}}{2 \Pi_{\xi}}\left(\frac{x}{L}\right)^{2}
$$

where the coefficients (derived by Maple computer algebra incorporated in Scientific WorkPlace from http://www.mackichan. com/) are

$$
\begin{aligned}
C_{o} & =\frac{\frac{\Pi_{f}}{2 \Pi_{\xi}} \sin \sqrt{\Pi_{\xi}}-\frac{\Pi_{f}}{\Pi_{\xi}}\left(\sqrt{\Pi_{\xi}}-\sin \sqrt{\Pi_{\xi}}\right)}{\sqrt{\Pi_{\xi}} \cos \sqrt{\Pi_{\xi}}-\sin \sqrt{\Pi_{\xi}}} \\
C_{1} & =\frac{\frac{-\Pi_{f}}{2 \sqrt{\Pi_{\xi}}} \cos \sqrt{\Pi_{\xi}}-\frac{\Pi_{f}}{\Pi_{\xi} \sqrt{\Pi_{\xi}}}\left(\cos \sqrt{\Pi_{\xi}}-1\right)}{\sqrt{\Pi_{\xi}} \cos \sqrt{\Pi_{\xi}}-\sin \sqrt{\Pi_{\xi}}} \\
A & =\frac{\frac{\Pi_{f}}{2 \Pi_{\xi}} \cos \sqrt{\Pi_{\xi}}-\frac{\Pi_{f}}{\bar{\Pi}_{\xi}}\left(1-\cos \sqrt{\Pi_{\xi}}\right)}{\sqrt{\Pi_{\xi}} \cos \sqrt{\Pi_{\xi}}-\sin \sqrt{\Pi_{\xi}}} \\
B & =\frac{\frac{-\Pi_{f}}{2 \Pi_{\xi}} \sin \sqrt{\Pi_{\xi}}-\frac{\Pi_{f}}{\Pi_{\xi}}\left(\sin \sqrt{\Pi_{\xi}}-\sqrt{\Pi_{\xi}}\right)}{\sqrt{\Pi_{\xi}} \cos \sqrt{\Pi_{\xi}}-\sin \sqrt{\Pi_{\xi}}}
\end{aligned}
$$

On the other hand, if we apply Swanson's partial-slip exit boundary conditions $y_{(x=L)}^{\prime \prime} \approx Y_{o}^{\prime \prime}$, so that the moment at the exit roller is negligible, the solution coefficients change to

$$
\begin{aligned}
& C_{o}= \frac{\frac{\Pi_{f}}{2 \Pi_{\xi}} \sin \sqrt{\Pi_{\xi}}+\left(Y_{o}^{\prime \prime} L-\frac{\Pi_{f}}{\Pi_{\xi}}\right)\left(\frac{\sqrt{\Pi_{\xi}}-\sin \sqrt{\Pi_{\xi}}}{\sqrt{\Pi_{\xi}}}\right)}{\sqrt{\Pi_{\xi}} \cos \sqrt{\Pi_{\xi}}-\sin \sqrt{\Pi_{\xi}} L} \\
& C_{1}=\frac{\frac{-\Pi_{f}}{2 \sqrt{\Pi_{\xi}}} \cos \sqrt{\Pi_{\xi}}+\left(Y_{o}^{\prime \prime} L-\frac{\Pi_{f}}{\Pi_{\xi}}\right)\left(\frac{\cos \sqrt{\Pi_{\xi}}-1}{\sqrt{\Pi_{\xi}}}\right)}{\sqrt{\Pi_{\xi}} \cos \sqrt{\Pi_{\xi}}-\sin \sqrt{\Pi_{\xi}} L} \\
& A=\frac{\frac{\Pi_{f}}{2 \Pi_{\xi}} \cos \sqrt{\Pi_{\xi}}+\left(Y_{o}^{\prime \prime} L-\frac{\Pi_{f}}{\Pi_{\xi}}\right)\left(\frac{1-\cos \sqrt{\Pi_{\xi}}}{\Pi_{\xi}}\right)}{\sqrt{\Pi_{\xi}} \cos \sqrt{\Pi_{\xi}}-\sin \sqrt{\Pi_{\xi}} L} \\
& B=\frac{\frac{-\Pi_{f}}{2 \Pi_{\xi}} \sin \sqrt{\Pi_{\xi}}+\left(Y_{o}^{\prime \prime} L-\frac{\Pi_{f}}{\Pi_{\xi}}\right)\left(\frac{\sin \sqrt{\Pi_{\xi}}-\sqrt{\Pi_{\xi}}}{\Pi_{\xi}}\right)}{\sqrt{\Pi_{\xi}} \cos \sqrt{\Pi_{\xi}}-\sin \sqrt{\Pi_{\xi}} L}
\end{aligned}
$$

While these are closed-form solutions, they are difficult to visualize; neglecting material stretching leads to conservative solutions which are much simpler. 


\section{Low-elasticity equilibrium solution}

If the elastic-stretching parameter $\Pi_{\xi}$ is very small, the governing Equation 36 can be reduced to

$$
y^{\prime \prime \prime \prime} \cong \frac{\mathbf{\Pi}_{f}}{L^{3}}
$$

If we apply Swanson's full-width-traction exit boundary condition $y_{(x=L)}^{\prime \prime}=0$, so that both sides of the web feed through the roller evenly, the solution is

$$
\frac{y}{L}=\Pi_{f}\left[\frac{1}{16}\left(\frac{x}{L}\right)^{2}-\frac{5}{48}\left(\frac{x}{L}\right)^{3}+\frac{1}{24}\left(\frac{x}{L}\right)^{4}\right]
$$

with an exit guiding angle

$$
\theta_{e x}=y_{(x=L)}^{\prime}=\frac{-\Pi_{f}}{48}
$$

and the lateral deflection profile shown in Figure 3. The maximum excursion of $y_{\max } / L=0.00542 \Pi_{f}$ occurs at $x / L=58 \%$.

On the other hand, if we apply Swanson's partial-slip exit boundary condition $y_{(x=L)}^{\prime \prime} \approx Y_{o}^{\prime \prime}$, so that the moment at the exit roller is negligible, the solution is

$$
\frac{y}{L}=\Pi_{f}\left[\frac{1}{16}\left(\frac{x}{L}\right)^{2}-\frac{5}{48}\left(\frac{x}{L}\right)^{3}+\frac{1}{24}\left(\frac{x}{L}\right)^{4}\right]+Y_{o}^{\prime \prime} L\left[-\frac{1}{4}\left(\frac{x}{L}\right)^{2}+\frac{1}{4}\left(\frac{x}{L}\right)^{3}\right]
$$

which gives us an exit guiding angle

$$
\theta_{e x}=y_{(x=L)}^{\prime}=\frac{1}{4} Y_{o}^{\prime \prime} L-\frac{1}{48} \Pi_{f}
$$

Evidently, the tension/stiffness ratio

$$
\text { tension/beam-parameter: } \Pi_{t / b} \triangleq \Pi_{f} / Y_{o}^{\prime \prime} L=b T_{a p p} L^{2} / E_{e f f} I
$$

determines the relative effect that any partial slip can have on the nature of the solution. When the value is $\Pi_{t / b}=8$, the deflection profile acquires an S-shape, the maximum amplitude is reduced, and the exit angle reversed, as shown by the dotted line in Figure 4, indicating that a partial-slip condition at the exit can be beneficial. However, for smaller values of $\Pi_{t / b}$, partial slip can reduce lateral control: the dotted line in the Figure 5 shows the lateral excursions with partial slippage when $\Pi_{t / b}=3.7$; it shows that partial-slip can be detrimental when $\Pi_{t / b}<3.7$

\section{LATERAL CONTROL}

One of the suggestions for control of the curvature within an air-flotation oven is to tilt every other air-bar-either the top set of bars, or else the bottom set of bars, or else both sets in opposite directions-in order to introduce a compensating curvature. However, we have noted that the lateral force due to the primary effect of normal pressure forces on the web depends only on the tilt of the web relative to our global co-ordinate system, not the tilt of the bars. On the other hand, we see that the web material's tension profile depends on the flying height $\left(Z-z_{b a r}\right)$. Therefore, if there is a significant contribution of web elasticity to the curvature, we can achieve some control by tilting air-bars.

Let us look at the case where all the top air-bars are tilted by an angle $\pm \alpha$ radians, and all the bottom air-bars are tilted by the same amount in the opposite 
direction, for a relative angle of $\pm 2 \alpha$. (The same result can be achieved by tilting only the top or only the bottom air-bars by $2 \alpha$.) The lateral forces on the web are unaffected, because they depend only on the tilt of the web itself; but the tension profile of the web depends on the relative tilt of web and air-bar, and therefore the moments in the web change. Revising Equation 23 accordingly,

$$
\text { Moment }=\frac{b^{3}}{12}\left[\frac{2 \ell^{2}}{\pi^{2} Z} \cdot y_{a i r}^{\prime \prime} \pm \alpha\right]\left[\frac{T_{a p p}-\left(\frac{\ell}{\pi}\right)^{2} \frac{d \tilde{F}}{d Z}}{Z}\right]
$$

we see that the effect of air-bar tilt is similar to the effect of the camber on moments. A tilt $\alpha$ with the proper sign can produce moments which assist in keeping the web straight.

Let us explore what this means for an inelastic, un-cambered (straight) web, for which $y^{\prime \prime}=y_{\text {air }}^{\prime \prime}$ : the lateral deflections will tend towards

$$
\begin{aligned}
{\left[\frac{2 \ell^{2}}{\pi^{2} Z} \cdot y_{a i r}^{\prime \prime} \pm \alpha\right] } & =0 \\
y^{\prime \prime} \ell & =\mp \alpha \cdot \frac{\pi^{2} Z}{2 \ell}
\end{aligned}
$$

giving us a quantitative indication of the nominal magnitude of steering effects from air-bar tilts. For the small values of $Z / \ell$ that we desire for robustness, only small control effects $y^{\prime \prime} \ell$ are possible. Conversely, if other considerations lead us to large web-amplitudes, tilt control on some or all bars might be promising.

\section{CONCLUSION}

1. The main effect of the sinusoidal shape of the web in an air-flotation oven is to reduce lateral bending stiffness. This reduction is directly proportional to the reduction of the extensional stiffness, insofar as the same "effective Young's modulus" enters into both MD extension and lateral bending. This makes extensional tests useful for judging lateral rigidity.

2. The same measures which raise extensional stiffness will improve lateral rigidity:

(a) Choice of air bars: bars which have been identified in our past experiments as having good flying-height "stiffness" will also improve extensional and lateral rigidity.

(b) Tension: increased tension will increase the flying-height "stiffness" as well as reduce sinusoidal amplitude.

(c) Air pressure, if it is matched by increased tension to maintain low flying height.

(d) Air-bar engagement: less engagement means a flatter web, with a moderate improvement in stiffness.

These four measures can be useful for controlling lateral-excursion troubles in ovens.

3. The lateral forces generated by the tilting of the web oppose the straightening effect of tension, indicating that air-support is inherently de-stabilizing with respect to lateral position. 
4. A remaining effect of tension is to increase the likelihood of good traction at the exit roller, and therefore a greater likelihood that the moderate-span full-width-traction boundary conditions apply, rather than partial-slip. As a result, increased tension can be counter-productive for straightening the web!

5. The main effect of air support is to reduce lateral moments in the web, eliminating slack regions and making longitudinal wrinkles less likely.

\section{FUTURE WORK}

Prof. Young B. Chang at Oklahoma State University is developing a lateraldeflection analysis which incorporates an air-cushion function $\mathfrak{F}$ based on groundeffect theory and confirmed by experiments [10].

\section{ACKNOWLEDGEMENTS}

This work was supported by the Web Handling Research Center at Oklahoma State University in Stillwater.

\section{REFERENCES}

1. Swanson, R.P., "Air-support conveyance of uniform and non-uniform webs," Proc. Second International Conference on Web Handling, Stillwater, OK, June 6-9, 1993, ISBN 0-935269-10-7, pp. 1-21.

2. Chang, Y.B., Swanson, R.P., \& Moretti, P.M., "Resiliency of an Air-Floated Web," Proc. Fifth International Conference on Web Handling, Stillwater, OK, June 6-9, 1999, ISBN 0-9654616-2-9, pp. 543-559.

3. Moretti, P.M., \& Chang, Y.B., "Coupling between Out-of-Plane Displacements and Lateral Stability of Webs in Air-Support Ovens," Proc. Third International Conference on Web Handling, Stillwater, OK, June 18-21, 1995, ISBN 9654616-0-2, pp. 338-347.

4. Pinnamaraju, R., "Measurement on air-bar/web interaction for the determination of lateral stability of a web in flotation ovens," MS thesis, MAE Dept., Oklahoma State University, Stillwater, OK, Dec. 1992.

5. Perdue, D.M., "Lateral stability investigation of air-bar and web interaction for use in flotation ovens," MS thesis, MAE Dept., Oklahoma State University, Stillwater, OK, Dec. 1993.

6. Nisankararao, S.K.V., "An experimental study of aerodynamic forces of air bars," MS thesis, MAE Dept., Oklahoma State University, Stillwater, OK, May 1994.

7. Chang, Y.B., \& Moretti, P.M., "Ground-effect theory and its application to air-flotation devices," Proc. Third International Conference on Web Handling, Stillwater, OK, June 18-21, 1995, ISBN 0-9654616-0-2, pp. 348-365.

8. Chang, Y.B., \& Moretti, P.M., "Aerodynamic characteristics of pressure-pad air bars," ASME International Mechanical Engineering Congress and Exposition, AD-Vol.53-2: 4th International Symposium on Fluid-Structure Interactions, Aeroelasticity, Flow-Induced Vibration and Noise, Volume II. Dallas, TX, Nov. 1997, pp. 3-9. 
9. Shelton, J.J., "Lateral dynamics of a moving web," Ph.D. Thesis, MAE Dept., Oklahoma State University, Stillwater, OK, July 1968.

10. Chang, Y.B., Swanson, R.P., \& Moretti, P.M., "Longitudinal and Out-ofPlane Stiffness of a Web in an Air-Flotation Oven," Proc. ASME, Noise Control and Acoustics Division - 1999, NCA-Vol.26, Nashville, Tennessee, Nov. 14-19, 1999, ASBN 0-7198-1637-0, pp. 435-443.

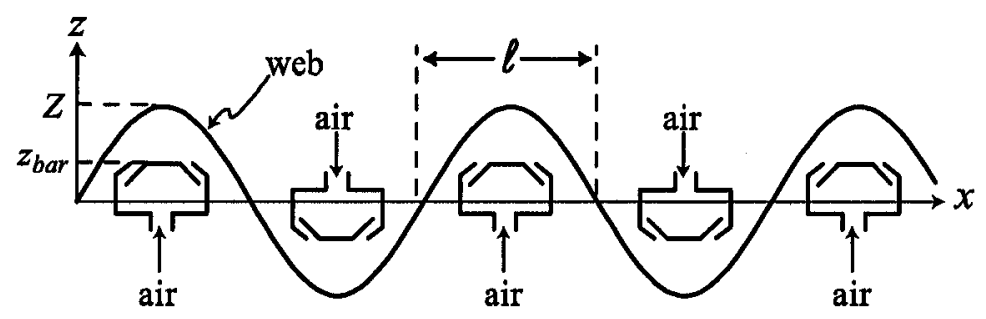

Figure 1: Nomenclature; the sine-wave is drawn with exaggerated amplitudeactual amplitudes are small, and $z_{b a r}$ may be negative.

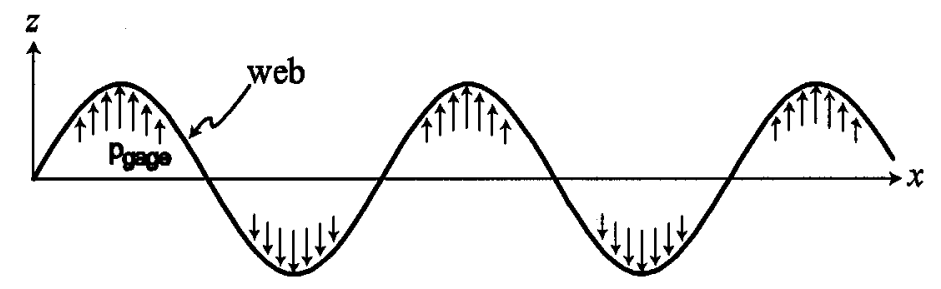

Figure 2: Relationship between web curvature and pressure distribution. 


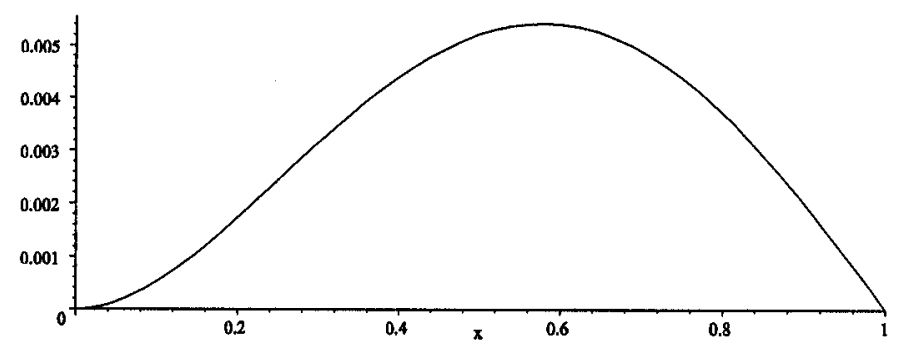

Figure 3: Deflection shape, $\mathrm{y} / \mathrm{L}$ versus $\mathrm{x} / \mathrm{L}$, from Equation 39, the low-elasticity equilibrium solution for a cambered web, with unit camber parameter (Equation $32)$.

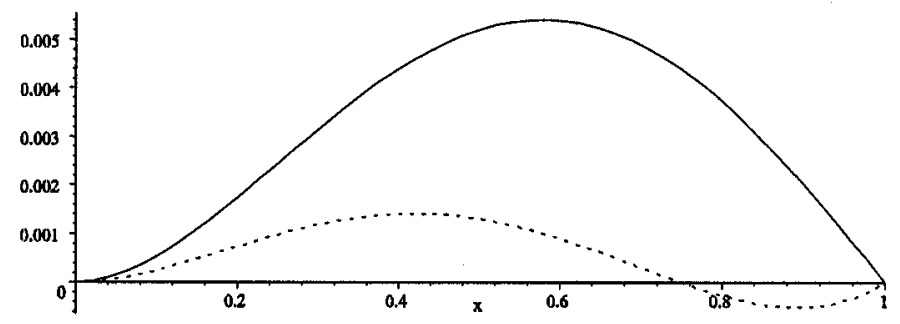

Figure 4: Deflection shape from Equation 41, the low-elasticity equilibrium solution for a cambered web, for partial-slip at the exit roller (dotted line), compared with full-width-traction at the exit (solid line), for a high value of 8 for the tension/beamparameter (Equation 43).

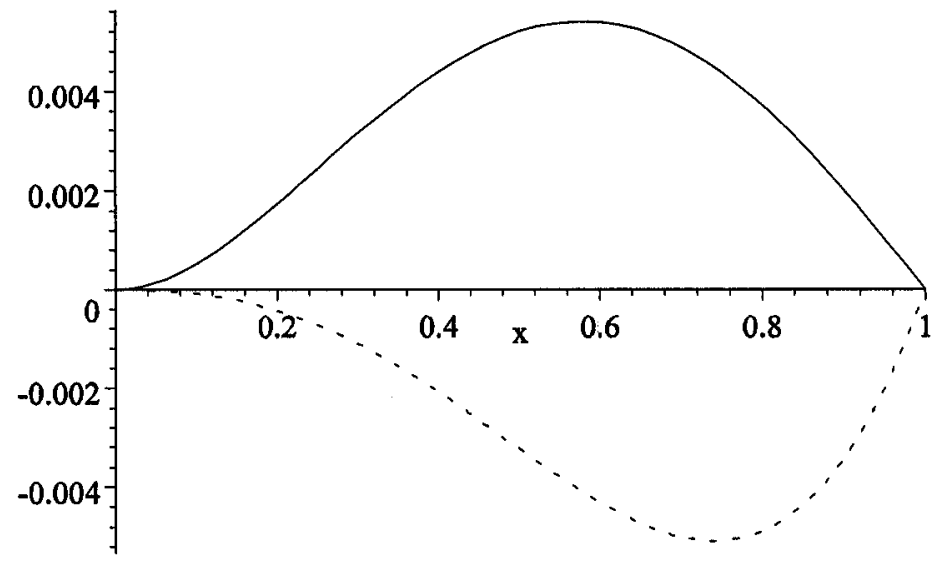

Figure 5: Deflection shape from Equation 41, the low-elasticity equilibrium solution for a cambered web, for partial-slip at the exit roller (dotted line), compared with full-width-traction at the exit (solid line), for a low value of 3.7 for the tension/beamparameter (Equation 43). 


\begin{tabular}{|l|l|}
\hline Name \& Affiliation & Question \\
\hline G. Homan - Westvaco & $\begin{array}{l}\text { I am wondering whether the illustration that you have } \\
\text { shown on your air flotation device is traditional in the } \\
\text { industry. My experience has been that in flotation ovens, } \\
\text { the web path is nearly straight and the length of the oven is } \\
\text { almost always the same as the web length. You can get the } \\
\text { geometry you have shown depending upon the tension vs. } \\
\text { the air velocity. I have seen this sort of thing from a } \\
\text { converting standpoint. }\end{array}$ \\
\hline Name \& Affiliation & $\begin{array}{l}\text { What you are saying is exactly right. I have drawn the } \\
\text { position of the air bars as overlapping, just to keep the } \\
\text { notation consistent. Commonly the overlap is a negative } \\
\text { number. I have also exaggerated the waviness to make the } \\
\text { picture easier to draw and look at. You'll notice that the } \\
\text { plots of lateral deflection are also exaggerated. What we } \\
\text { see in the equations is that you have to keep the waviness } \\
\text { very small, because it is the waviness that leads you into } \\
\text { trouble. The small amplitude is what makes the first-order } \\
\text { analysis work so well. The other end of the spectrum is the } \\
\text { video-graphic film air reverser situation, which we can also } \\
\text { handle with this type of analysis, but because the turning } \\
\text { angles are so large, we'd have to reformulate the equations. } \\
\text { We would only want to use a continuum model if there } \\
\text { were at least five to seven reversers; otherwise it's better to } \\
\text { handle them one by one. }\end{array}$ \\
\hline Question \\
\hline P. OSU & $\begin{array}{l}\text { From a realistic standpoint, I see a lot of misalignment of } \\
\text { elements and if maintenance becomes shoddy, the elements } \\
\text { can begin to be filled with various types of debris, which } \\
\text { really upsets the system. }\end{array}$ \\
\hline Answer \\
\hline Name \& Affiliation
\end{tabular}

